\title{
"Organoid-in-a-column" coupled on-line with liquid chromatography-mass spectrometry
}

Stian Kogler ${ }^{1,2}$, Sean Harrison ${ }^{1}$, Aleksandra Aizenshtadt ${ }^{1}$, Frøydis Sved Skottvoll ${ }^{1,2}$, Shadab Abadpour $^{1,3}$, Stefan Krauss ${ }^{1}$, Hanne Scholz ${ }^{1,3}$, Gareth Sullivan ${ }^{1,4,5,6,7}$, Elsa Lundanes ${ }^{2}$, Steven Ray Wilson ${ }^{1,2} *$

${ }^{1}$ Hybrid Technology Hub-Centre of Excellence, Institute of Basic Medical Sciences, Faculty of Medicine, University of Oslo, Oslo, Norway

${ }^{2}$ Department of Chemistry, University of Oslo, Oslo, Norway

3 Department of Transplant Medicine, Institute for Surgical Research, Oslo University Hospital, Oslo. Norway

${ }^{4}$ Norwegian Center for Stem Cell Research, University of Oslo, Oslo, Norway

${ }^{5}$ Department of Molecular Medicine, Institute of Basic Medical Sciences, University of Oslo, Oslo, Norway

${ }^{6}$ Institute of Immunology, Oslo University Hospital, Oslo, Norway

${ }^{7}$ Department of Pediatric Research, Oslo University Hospital, Oslo, Norway

* Corresponding author: Professor Steven Ray Wilson, stevenw@kjemi.uio.no, +47 97010953. Full address: Department of Chemistry, University of Oslo, Post Box 1033, Blindern, NO-0315 Oslo, Norway. https://orcid.org/0000-0002-9755-1188 


\begin{abstract}
Organoids are stem-cell derived "mini organs" and are emerging tools for e.g. developmental biology and drug development. We have packed empty liquid chromatography column housings (micro format) with induced pluripotent stem cell (iPSC) derived hepatic organoids, and coupled these "organoid-in-a-column" units directly to liquid chromatography-mass spectrometry. The system has been charged with heroin, followed by on-line monitoring of the drug's phase 1 metabolism. Using a C4 stationary phase for reverse phase liquid chromatography, small molecule measurements were not significantly affected by liver organoid medium matrix (consisting of e.g. fetal bovine serum). Enzymatic metabolism of heroin was reliably detected using a triple quadrupole instrument over a period of two days, demonstrating increased metabolite production over time and serving as a proof-of-concept for on-line coupling of organoids and mass spectrometry.
\end{abstract}

Keywords: Organoid; Induced pluripotent stem cells; On-line; Liquid chromatography; Mass Spectrometry; Metabolism 


\section{Introduction}

Drug discovery and development is an extremely costly process, and the number of new drugs reaching market per billion dollars spent on drug development is steadily declining [1]. Moreover, many new drugs can be ineffective (or even toxic) to a large portion of target patients [2]. Key bottlenecks of efficient drug development include the limited predictive value of traditional cell cultures and animal models regarding human trials [3]. Furthermore, reliable technologies for predicting personalized drug metabolism, a key issue in toxicology, are lacking.

Organoids are emerging tools for drug discovery and development. These miniature 3D models of actual organs can be grown from human stem cells, and therefore carry potential for being relevant and personalized testing materials [4].

Typically, organoids feature multiple cell types specific of the organ they mimic, and have biological properties and morphologies that (to varying degrees) resembling the model organ [5]. Current liver organoids contain parenchymal cells and endothelial cells in a rather unorganized spacial arrangement. In iPS derived liver organoids, the parenchymal cells (hepatocytes) elicit an immature fetal phenotype [6]. Nevertheless, within these limitations, liver organoids can be tools for assessing aspects of drug metabolism, in particular phase 1 metabolism, and toxicity.

To analyze the metabolic capability of organoids, liquid chromatography (LC-MS) is the method of choice. Mass spectrometric analysis of organoids is today commonly performed indirectly, or "off-line", i.e. samples are collected and handled semi-manually prior to MS [79]. This process can be time consuming and prone to variations based on sample handling. Direct on-line analysis of organoids could offer the advantage of increased automation and precision and hence reduced sample variation [10].

We here describe the first on-line system for detecting organoid-derived metabolites of drugs using MS, also featuring liquid chromatographic separation. The system is related to that described by van Midwoud and co-workers [11], who performed on-line analysis of drug metabolism of liver slices, using custom-built microfluidic chips and UV detection. However, we have simplified the work flow, created an incubation device (essentially an empty LC 
separation column which has been filled with organoids) without the need for specialized parts and coupled the system directly to a MS. Consisting of simple parts and standard commercial instrumentation, the here presented system is a scalable on-line MS analysis platform for monitoring drug metabolism in liver organoids upon drug challenges.

\section{Experimental}

\section{Consumables and hardware}

Stainless steel (SS) unions, reducing unions (1/16" to $\left.1 / 32^{\prime \prime}\right)$, SS ferrules and nuts (all for $1 / 16$ " tubing and for $1 / 32$ " tubing), SS tubing (1/32" outer diameter (OD), 0.020 " inner diameter (ID) and $0.005^{\prime \prime}$ ID) and 1/16" SS screens ( $1 \mu \mathrm{m}$ pores) were purchased from $\mathrm{VICl}$ Valco (Schenkon, Switzerland).

SST Vipers $(130 \mu \mathrm{m} \times 650 \mathrm{~mm}$ ) were purchased from Thermo Fisher Scientific (Waltham, MA, USA). Polytetrafluoroethylene (PTFE, Teflon) tubing (1/16" OD, 1/32" ID) was purchased from Darwin microfluidics (Paris, France). A chromatographic column (1 $\mathrm{mm} \times 5 \mathrm{~cm}$ ) packed with Kromasil C4 (3.5 $\mu \mathrm{m}, 100 \AA$ ) was purchased from Teknolab (Ski, Norway). Luer lock syringes (10-20 mL) were purchased from B. Braun Melsungen AG (Hessen, Germany).

\section{Reagents and solutions}

Formic acid ( $\geq 98 \%$ ) was purchased from Merck (Darmstadt, Germany). Water (LC-MS grade) and acetonitrile (ACN, LC-MS grade) were purchased from VWR International (Oslo, Norway).

For liquid chromatography (LC) Mobile phase (MP) reservoir A contained $0.1 \%$ formic acid in HPLC water (v/v). MP reservoir B contained ACN/HPLC water/FA (90/10/0.1\%, v/v/v).

PhD Inger Lise Bogen (Oslo University Hospital) supplied solutions of heroin, 6-MAM, morphine. Fetal bovine serum-free medium and L15 base medium was prepared according to [12], and is hereafter referred to as organoid medium.

Organoids 
iPSC line AG27 was differentiated to form liver organoids as described by Harrison et al. (manuscript in preparation). iPSC cell lines HPSI1213i-babk_2 and HPSI0114i vabj_3 were differentiated toward liver organoids using a modification of the protocol by Ang et al. [13], by Aizenshtadt et al. (manuscript in preparation).

\section{Instruments}

The Dionex UltiMate 3000 UHPLC system and the TSQ Vantage MS with the HESI-II ion source were purchased from Thermo Fisher Scientific. The syringe pump (AL-1000) was bought from World Precision Instruments (Sarasota, FL, USA). A 2-position 10-port valve (for 1/32", C82X-6670ED) was purchased from VICl Valco. A SUB Aqua 5 Plus water bath was purchased from Grant Instruments (Cambridge, UK).

\section{Organoid-in-a-column}

For fabrication of the column housing, an approximately $5 \mathrm{~cm}$ long piece of Teflon/PFTE tubing (1/16" OD, 1/32" ID) was cut and assembled with nuts and SS ferrules. To one end of the tube, a union with a $1 \mu \mathrm{m}$ screen ( $\mathrm{VICl}$ Valco) was connected.

A volume of 1-22 $\mathrm{mL}$ of medium containing organoids was then filled into a Luer-lock syringe, and the syringe was subsequently connected to the open end of the column. With the syringe mounted on a syringe pump, the medium was pumped through the column housing at a flow rate of $100 \mu \mathrm{L} / \mathrm{min}$. Once the entire contents of the syringe was passed through the column, the inlet was fitted with a screen and a union. A schematic of an organin-a-column is shown in Figure 1.

For idle conditions, a new syringe was filled with fresh organoid medium and connected to the organ-in-a-column, so that the flow was the opposite way as during filling. The pump was set to $0.20 \mu \mathrm{L} / \mathrm{min}$. After filling, the organoid column was submerged in a water bath at $37^{\circ} \mathrm{C}$. 


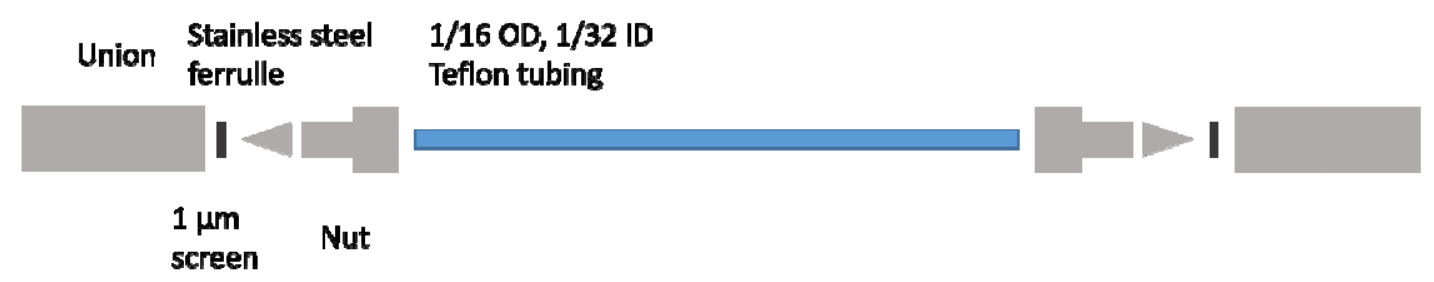

Figure 1. Column housing for "organoid-in-a-column".

Organoid-in-a-column coupled with liquid chromatography-mass spectrometry

The pump/syringe/organoid-in-a-column system described above was connected to LC-MS instrumentation. Eluate from the column was transported to a valve system for fractionation. The valve system contained two loops, which were filled sequentially. As one loop was being filled, the contents of the other loop were transferred to a $5 \mathrm{~cm} \times 1 \mathrm{~mm} \mathrm{C4}$ LC column for separation prior to MS detection. The $20 \mu \mathrm{L}$ loops were overfilled with an additional $5 \mu \mathrm{L}$ to ensure that any LC solvent left in the loop would be flushed off. See Figure 2 for a schematic of the setup. For studying heroin metabolism, $100 \mu \mathrm{M}$ solutions in medium were pumped at $0.20 \mu \mathrm{L} / \mathrm{min}$, with fractionation each $120^{\text {th }}$ minute. See Table 1-3 for LC-MS conditions.

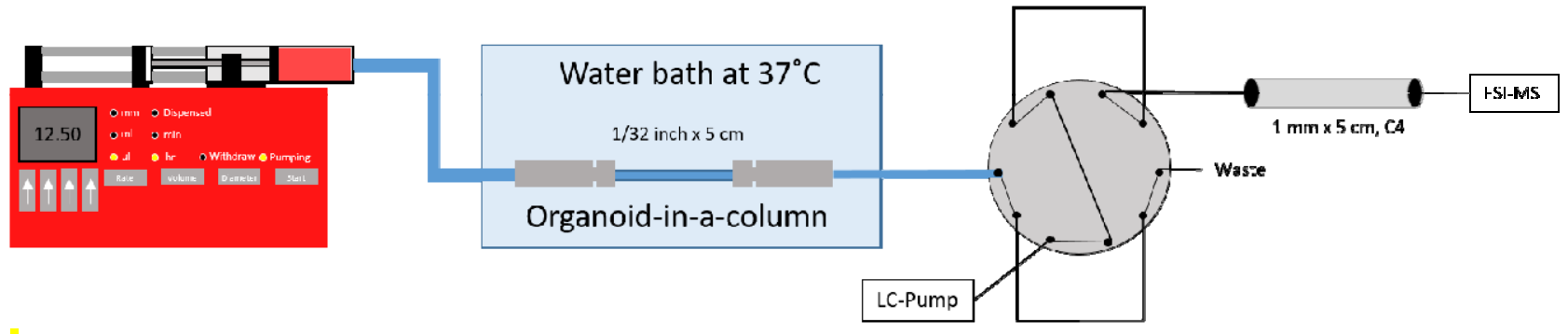

Figure 2. Illustration of "organoid-in-a-column" coupled on-line with liquid chromatography-mass spectrometry. See Experimental section for more details.

Table 1. LC-gradient for on-line studies of "organoid-in-a-column" metabolism of heroin.

\begin{tabular}{|l|l|l|l|}
\hline Time, $\min$ & Flow, $\boldsymbol{\mu L} / \mathbf{m i n}$ & \%B & Purpose \\
\hline 0 & 50 & 3 & Separation \\
\hline 20 & 50 & 20 & \\
\hline 20 & 50 & 80 & Wash \\
\hline 60 & 50 & 80 & \multirow{2}{*}{ Re-equilibration } \\
\hline 60 & 50 & 3 & \\
\hline 70 & 50 & 3 & Save MP \\
\hline 70 & 0 & 3 & \\
\hline
\end{tabular}




\begin{tabular}{|l|l|l|l|}
\hline 110 & 0 & 3 & \\
\hline 110 & 50 & 3 & Re-equilibration \\
\hline 120 & 50 & 3 & \\
\hline
\end{tabular}

Table 2. Multiple reaction monitoring (MRM) parameters used for the detection of heroin and its phase 1 metabolites.

\begin{tabular}{|l|l|l|l|}
\hline Molecule & Parent ion, $\mathbf{m} / \mathbf{z}$ & Product ion, $\mathbf{m} / \mathbf{z}$ & Collision energy, eV \\
\hline Heroin & 370 & 272 & 30 \\
\hline 6-MAM & 328 & 211 & 25 \\
\hline Morphine & 286 & 201 & 20 \\
\hline
\end{tabular}

Table 3. General MS parameters for detection of heroin, 6-MAM and morphine

\begin{tabular}{|l|l|}
\hline Parameter & Value \\
\hline Capillary temperature & $300.0^{\circ} \mathrm{C}$ \\
\hline Vaporizer temperature & $200.0^{\circ} \mathrm{C}$ \\
\hline Sheath gas pressure & 20.0 \\
\hline lon Sweep gas pressure & 0.0 \\
\hline Auxillary air flow & 5.0 \\
\hline Spray voltage & Pos: 3000.0 V Neg: $0.0 \mathrm{~V}$ \\
\hline Collision gas pressure & 1.0 mTorr \\
\hline
\end{tabular}

\section{Live/Dead staining}

Six days after the final drug metabolism experiments, the organoid-in-a-column was flushed with phosphate-buffered saline (PBS) $\mathrm{pH} 7.4$ to remove the medium. The column was dismantled and the content was collected. Organoids that were stuck to the filters were scraped off and transferred to a glass slide and excess PBS was removed. The organoids were then stained with Live/Dead Cell Double Staining Kit suitable for fluorescence from Sigma Aldrich in the following manner: $100 \mu \mathrm{L}$ of the staining solution (5 mL PBS, $10 \mu \mathrm{L}$ solution A and $5 \mu \mathrm{L}$ solution B) were pipetted onto the organoids. A cover slide was placed on top and the organoids were then incubated at $37^{\circ} \mathrm{C}$ for 15 minutes prior to microscopy.

\section{Results and Discussion}

Organoids were packed in liquid chromatography column housings. A micro LC format housing was chosen (1/32" ID and $5 \mathrm{~cm}$ length, see Figure 1). In separation science, such sizes, belonging to the category "miniaturized LC", are associated with low radial dilution and suited for small sample analysis [14]. Medium containing organoids was syringe-pumped into an open LC column housing at flow rates at lower/mid $\mu \mathrm{L} / \mathrm{min}$ ranges (depending on 
organoid sizes). After the on-line measurements, organoids were flushed from the column using the same instrumentation; Pre-loading and post-flushing inspection of the organoids by microscopy revealed tissue intactness.

The low pressure "organoid-in-a-column" was coupled with high-pressure LC through a fractionation valve set-up. LC-MS is generally operated at high pressures e.g. 50-400 bars, which is incompatible for organoids. A SS-valve was used to collect and send fractions to the LC-MS system, not unlike that used for two-dimensional LC separations [15]. The valve system set-up also isolated the organoids from the high-pressure analysis system (see Figure 2).

A C4 stationary phase for liquid chromatography was suited for metabolite and medium analysis. Organoid medium is highly complex and contains considerable amounts of e.g. albumin. Sample complexity and presence of proteins can cause unpredictable chromatographic performance. A C4 stationary phase (considered relatively compatible with proteins) allowed for repeatable chromatography of medium spiked with drugs and metabolites (see Figure 3). In addition, we speculate that the rather low pore size of the particles employed $(100 \AA)$ aided in separating macromolecules from small drugs via size exclusion. It should however be mentioned that the ion source of the mass spectrometer would become contaminated with the complex mixture, calling for extra attention to maintenance and/or additional source protections. Serum-free medium could be used to avoid the uncontrollable composition of fetal bovine serum (FBS), but will contain high amounts of proteins from supplements and other necessary medium components. Hence, proteins are an inevitable part of medium and need to be dealt with accordingly.

The mobile phase composition was also a key parameter regarding robustness, as e.g. methanol as an organic modifier was associated with column clogging and poor performance when chromatographing the organoid medium. 


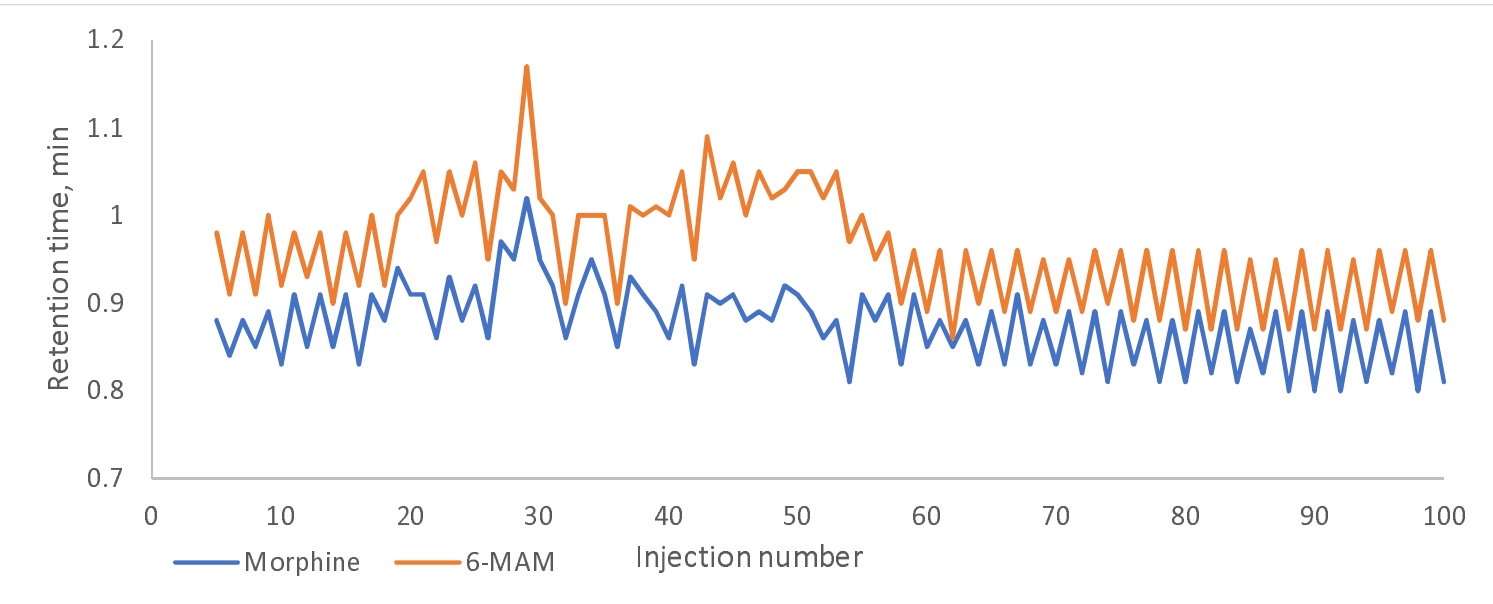

Figure 3. Retention time variability of heroin metabolites using "organoid-in-a-column" coupled on-line with liquid chromatography-mass spectrometry (100 injections). Zig-zag pattern due to a bridge tubing, creating slightly longer distance from one sample loop to the MS, thereby extending the retention time.

The on-line system was suited for observing enzyme-based drug metabolism of organoids. Heroin was chosen as a model compound as it is a well-studied drug with a simple and wellunderstood metabolism [16-18]. It was believed that this would simplify the qualitative measurement of its metabolites, as established methods are available. In initial experiments, organoid medium $+1 \mu \mathrm{M}$ heroin was applied to the organoid-in-a-column at a flow rate of 1 $\mu \mathrm{L} / \mathrm{min}$. Heroin and 6-MAM were detected, but the latter was likely detected due to spontaneous decomposition of heroin to 6-MAM [17]. For evaluating enzymatic drug metabolism of the "organoid-in-a-column", a lower flow rate of $12.5 \mu \mathrm{L} / \mathrm{h}$ was used with a heroin concentration of $100 \mu \mathrm{M}$. A total time of 120 minutes per injection was used. Ample signals were obtained for heroin and metabolites 6-MAM and morphine for each fraction analyzed by LC-MS. The final extracted chromatogram for morphine is shown in Figure 4. For 6-MAM and morphine, the peak height and area increased substantially over the course of 22 injections (Figure 5). The concentration of 6-MAM was expected to increase until all heroin was consumed, as it was added to the medium and stored at room temperature during the experiment. This point was reached after eight injections (corresponding to 16 hours). The concentration of morphine, which only is formed through enzymatic activity, increased beyond this point, pointing towards an upregulation either of the metabolizing enzymes or the transportation of drugs and metabolites in and out of the cells or both. 
bioRxiv preprint doi: https://doi.org/10.1101/2020.09.08.282756; this version posted September 19, 2020. The copyright holder for this preprint (which was not certified by peer review) is the author/funder, who has granted bioRxiv a license to display the preprint in perpetuity. It is made available under aCC-BY 4.0 International license.

Expectedly, the kinetics were slower than that observed with e.g. liver microsomes and S9 fraction experiments. The retention time of the analytes remained stable throughout the experiment, see Figure 6.

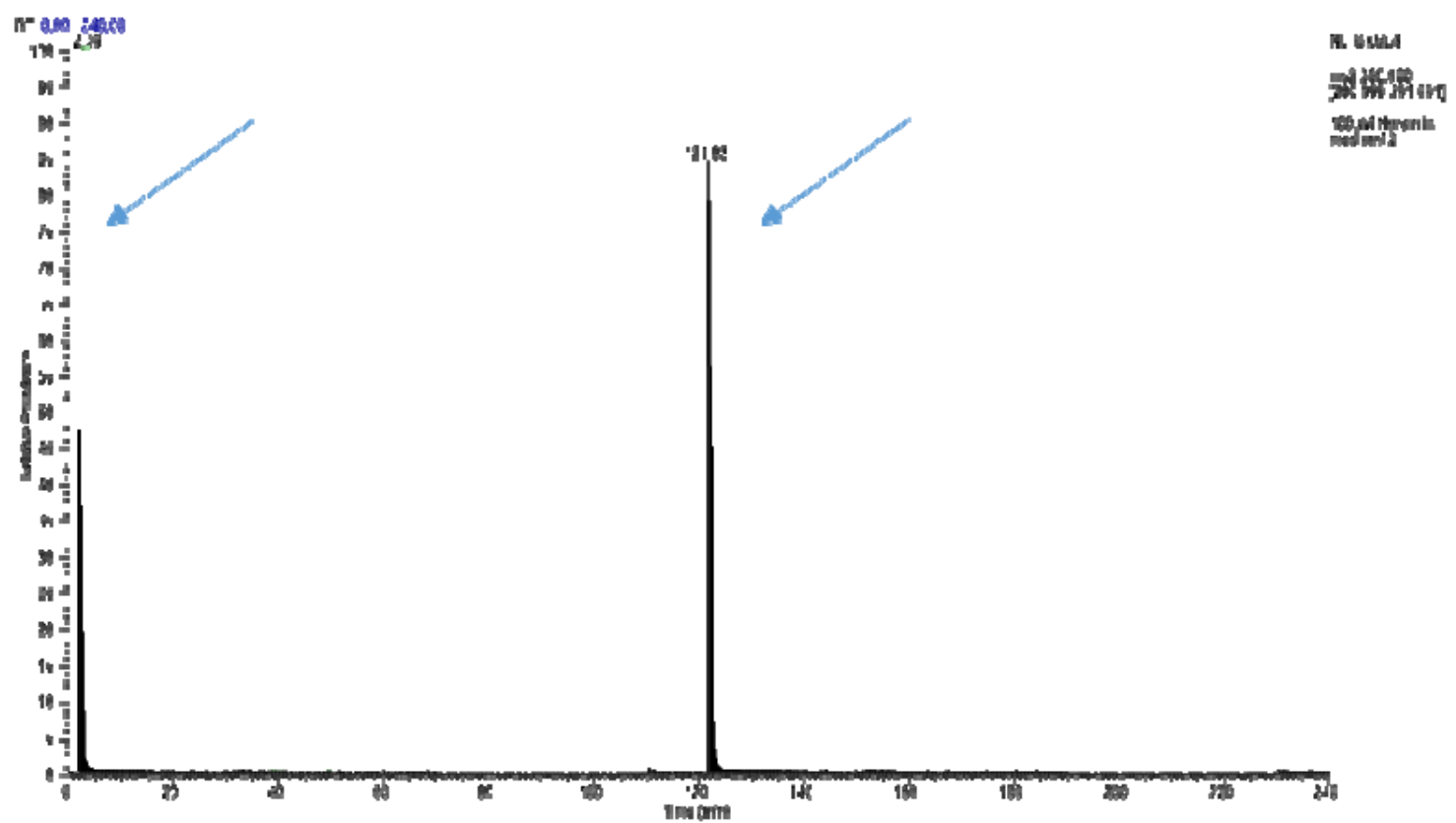

Figure 4. LC-MS chromatogram of the two morphine peaks $(\mathrm{m} / \mathrm{z} 286$ ] $\mathrm{m} / \mathrm{z} 201)$ derived from organoid-in-acolumn exposed to heroin. Injections were made at $t=0$ and $t=120 \mathrm{~min}$.

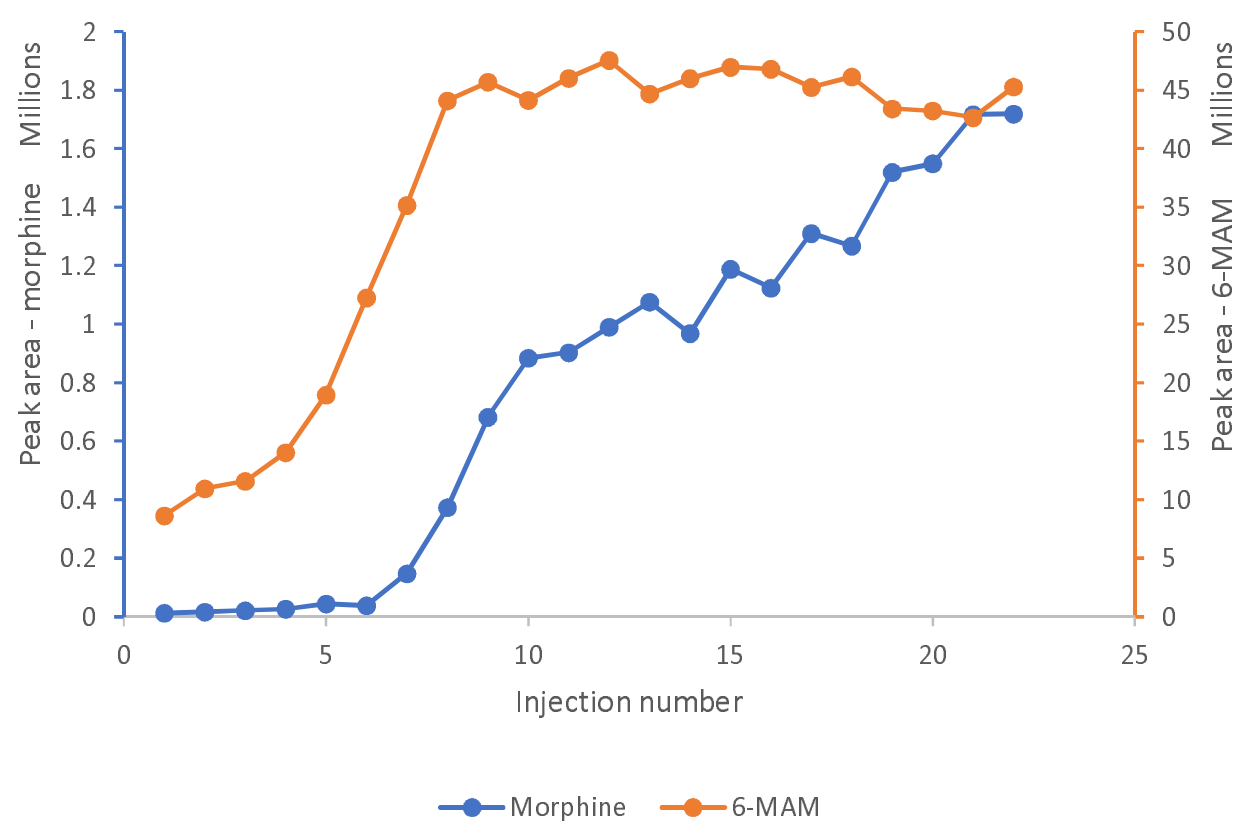

Figure 5. Peak area as a plot of injection number. Peak area of morphine and 6-MAM was increasing substantially throughout 22 injections. Morphine concentration increased even after 6-MAM reached a plateau. 


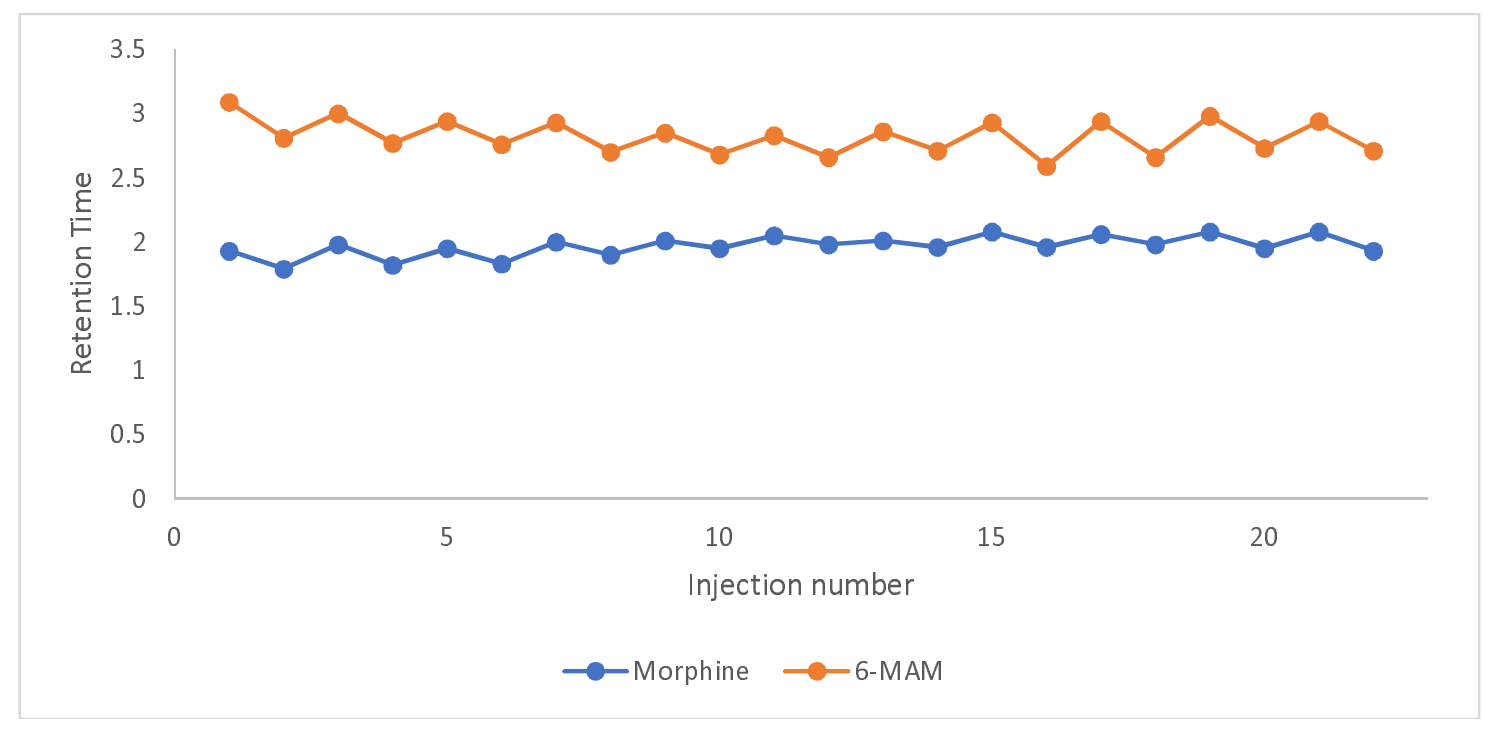

Figure 6. Plot of retention times for morphine and 6-MAM from the 22 injections made. Retention time remained stable-Zig-zag pattern due to slightly longer distance to MS from one sample loop.

Organoids were able to survive the conditions of the experiment. After the on-line measurements of heroin metabolism, the organoid-in-a-column was left under perfusion of medium for six days. Then, the organoid column was taken apart and its content collected for staining. A live/dead staining kit was used to stain live and dead cells (calcein acetoxymethyl/propidium iodide). Figure 7 demonstrates that organoids could survive the fairly harsh settings applied, indicating that the developed system may work for long-term cultivation under appropriate conditions.

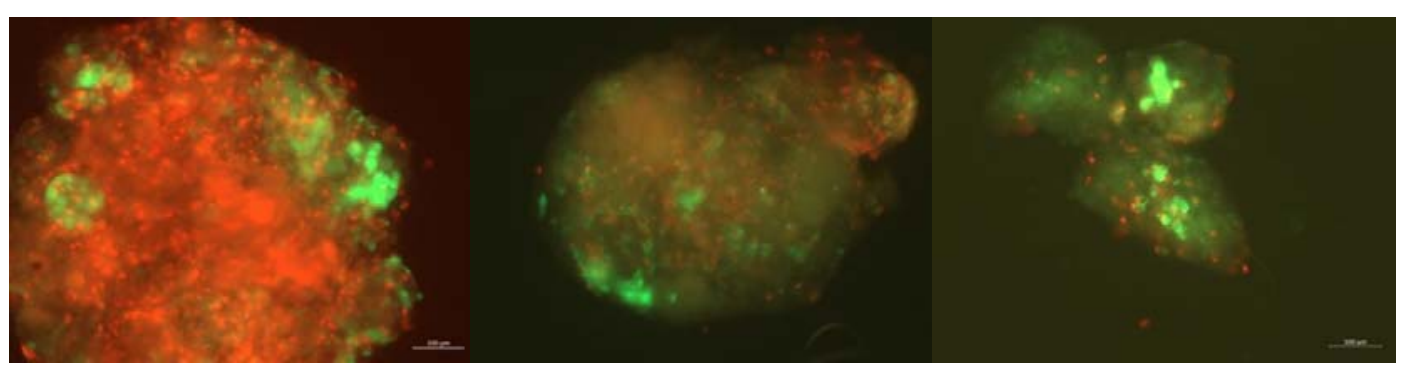

Figure 7. Live/dead staining of some organoids after organoid-on-a-columns experiments. Organoids stained could survive (middle, right) even after exposure to high concentrations of heroin and left under perfusion of medium without oxygenation for more than 7 days. However, not all organoids survived (left). Scale bar is 100 $\mu \mathrm{m}$. 
A set-up for measuring organoid metabolism shows promise through a quite simple concept, namely "packing" organoids in a LC column housing, which is readily adaptable for on-line analysis. Initially, we probed the use of commercial organ-on-a-chip housings (e.g. ones with PDMS-based materials), but with little success. During the time of this work, most commercial housings have been designed for e.g. confocal microscopy experiments, with little attention to coupling options, fragility, and potential leakages that can occur in microfluidic coupling. We also experimented with in-house developed chip designs, which however, had issues related to poor retention of the organoids. LC column housings, on the other hand, are specifically designed for avoiding leakages and simple "packing", and come in a variety of diameters and lengths, with readily available fittings.

The "organoid-in-a-column" variant allowed for automated LC-MS action, with a proof-ofprinciple shown with heroin metabolism. We will expand the technology to systematically test phase 1 metabolic pathways, e.g. of CYP-activity tell-tale molecules, e.g. diclofenac and imidazole.

We are exploring the addition of various on-line sample clean up steps, e.g. coupling with electro-membrane extraction (EME) [19, 20] or solid phase extraction (SPE) [10]. This may allow for cleaner extracts to enter the LC-MS system. We believe this will be a necessity for an upscaled version or when performing thousands of injections on a system. We therefore view the organoid-in-a-column-LC-MS system as an early stage concept and invite our colleagues in the field to investigate the coupling of mass spectrometry and organoids.

To assess the crosstalk between organs, we are currently investigating organoid-in-a-column coupling of two different tissue types in series, i.e. as a two-column system of for example islets of Langehans and liver.

As the size and with it the number of cells can vary greatly between organoids, proper standardization of loading is difficult to achieve. While larger organoids should give a theoretical higher metabolizing ability, cells in smaller organoids, to a larger degree, will be directly exposed to the drug. Which approach is favored, remains to be tested.

As it was difficult to standardize the numbers of organoids in each column, some were loaded with too many organoids. This caused the loaded organoids to form a plug that 
eventually clogged the system. Therefore, future systems will contain support material, which will aid in the distribution of single organoids throughout the column. Such support material could be beads or porous "sponges" of glass, silica, matrigel, alginate, PDMS or other materials and may be engineered with additives to work as sensors for e.g. $\mathrm{pO}_{2}, \mathrm{pCO}_{2}$, temperature, $\mathrm{pH}$, or even selective sensing of compounds.

Organoid-in-a-column, is a simple approach for testing drug metabolism without the need for highly specialized parts. The technology should therefore be readily available to anyone in need of an in vitro system for the metabolism studies of tissue. The addition of an on-line sample clean-up step should make the technology scalable for high throughput applications such as large-scale drug testing.

\section{Acknowledgements}

This work was supported by the Research Council of Norway through its Centre of Excellence scheme, project number 262613. The work was also supported by the Olav Thon Foundation. Financial support from UiO:Life Science is also gratefully acknowledged.

SRW would like to give special thanks to Billy Conway, Mark Sandman, Dana Colley, Jerome Deupree for inspiration in choice of metabolites to monitor.

\section{References}

[1] J.A. DiMasi, H.G. Grabowski, R.W. Hansen, Innovation in the pharmaceutical industry: new estimates of $R \& D$ costs, Journal of health economics, 47 (2016) 20-33

[2] H. Olson, G. Betton, D. Robinson, K. Thomas, A. Monro, G. Kolaja, P. Lilly, J. Sanders, G. Sipes, W. Bracken, Concordance of the toxicity of pharmaceuticals in humans and in animals, Regulatory Toxicology and Pharmacology, 32 (2000) 56-67

[3] A. Skardal, T. Shupe, A. Atala, Organoid-on-a-chip and body-on-a-chip systems for drug screening and disease modeling, Drug discovery today, 21 (2016) 1399-1411

[4] S.N. Bhatia, D.E. Ingber, Microfluidic organs-on-chips, Nature biotechnology, 32 (2014) 760-772

[5] M.A. Lancaster, J.A. Knoblich, Organogenesis in a dish: modeling development and disease using organoid technologies, Science, 345 (2014) 1247125

[6] T. Takebe, B. Zhang, M. Radisic, Synergistic engineering: organoids meet organs-on-achip, Cell stem cell, 21 (2017) 297-300

[7] C. Dorrell, B. Tarlow, Y. Wang, P.S. Canaday, A. Haft, J. Schug, P.R. Streeter, M.J. Finegold, L.T. Shenje, K.H. Kaestner, The organoid-initiating cells in mouse pancreas and liver are phenotypically and functionally similar, Stem cell research, 13 (2014) 275-283 
[8] B.J. Tauro, D.W. Greening, R.A. Mathias, S. Mathivanan, H. Ji, R.J. Simpson, Two distinct populations of exosomes are released from LIM1863 colon carcinoma cell-derived organoids, Molecular \& Cellular Proteomics, 12 (2013) 587-598

[9] S.F. Boj, C.-I. Hwang, L.A. Baker, I.I.C. Chio, D.D. Engle, V. Corbo, M. Jager, M. PonzSarvise, H. Tiriac, M.S. Spector, Organoid models of human and mouse ductal pancreatic cancer, cell, 160 (2015) 324-338

[10] M. Rogeberg, H. Malerod, H. Roberg-Larsen, C. Aass, S.R. Wilson, On-line solid phase extraction-liquid chromatography, with emphasis on modern bioanalysis and miniaturized systems, Journal of pharmaceutical and biomedical analysis, 87 (2014) 120-129

[11] P.M. van Midwoud, J. Janssen, M.T. Merema, I.A. de Graaf, G.M. Groothuis, E. Verpoorte, On-line HPLC analysis system for metabolism and inhibition studies in precisioncut liver slices, Analytical Chemistry, 83 (2010) 84-91

[12] S. Mathapati, R. Siller, A.A. Impellizzeri, M. Lycke, K. Vegheim, R. Almaas, G.J. Sullivan, Small-molecule-directed hepatocyte-like cell differentiation of human pluripotent stem cells, Current protocols in stem cell biology, 38 (2016) 1G. 6.1-1G. 6.18

[13] L.T. Ang, A.K.Y. Tan, M.I. Autio, S.H. Goh, S.H. Choo, K.L. Lee, J. Tan, B. Pan, J.J.H. Lee, J.J. Lum, A roadmap for human liver differentiation from pluripotent stem cells, Cell reports, 22 (2018) 2190-2205

[14] S.R. Wilson, T. Vehus, H.S. Berg, E. Lundanes, Nano-LC in proteomics: recent advances and approaches, Bioanalysis, 7 (2015) 1799-1815

[15] D.R. Stoll, P.W. Carr, Two-dimensional liquid chromatography: a state of the art tutorial, in, ACS Publications, (2017).

[16] U. Boerner, S. Abbott, R.L. Roe, The metabolism of morphine and heroin in man, Drug metabolism reviews, 4 (1975) 39-73

[17] L.M. Kamendulis, M.R. Brzezinski, E.V. Pindel, W.F. Bosron, R.A. Dean, Metabolism of cocaine and heroin is catalyzed by the same human liver carboxylesterases, Journal of Pharmacology and Experimental Therapeutics, 279 (1996) 713-717

[18] D.A. Barrett, A.L. Dyssegaard, P.N. Shaw, The effect of temperature and $\mathrm{pH}$ on the deacetylation of diamorphine in aqueous solution and in human plasma, Journal of pharmacy and pharmacology, 44 (1992) 606-608

[19] F.S. Skottvoll, F. Hansen, S. Harrison, I.C.S. Boger, A. Mrsa, M.S. Restan, M. Stein, E. Lundanes, S. Pedersen-Bjergaard, A. Aizenshtadt, Electromembrane extraction and mass spectrometry for liver organoid drug metabolism studies, BioRxiv, (2020)

[20] F.A. Hansen, D. Sticker, J.r.P. Kutter, N.J. Petersen, S. Pedersen-Bjergaard, Nanoliterscale electromembrane extraction and enrichment in a microfluidic chip, Analytical chemistry, 90 (2018) 9322-9329 